\title{
Effect of kenaf seed oil from different ways of extraction towards ovarian cancer cells.
}

\begin{abstract}
Kenaf (Hibiscus cannabinus) from the family of Malvaceae is a valuable fibre plant native to India and Africa. Kenaf is composed of various active components including tannins, saponins, polyphenolics, alkaloids, essential oils and steroids. It has been used to treat bruises, bilious conditions, fever and puerperium. Nevertheless, the anti-cancer properties of kenaf seed oil have not yet been investigated. In this study, kenaf seed oils obtained by Sonication, Soxhlet and supercritical carbon dioxide fluid extraction (SFE) with 9 different combinations of pressure (bars) and temperature $\left({ }^{\circ} \mathrm{C}\right)(200 / 40,200 / 60,200 / 80,400 / 40$, $400 / 60,400 / 80,600 / 40,600 / 60$ and 600/80) were investigated for the cytotoxicities. All the oils were cytotoxic towards ovarian cancer (CaOV3) and colon cancer (HT29) cell lines in a dose dependent manner as detected by using the MTT assay and trypan blue dye exclusion method. Oil from Sonication was the most cytotoxic towards CaOV3 cell line. Treated cells exhibited characteristics of apoptosis such as chromatin condensation and nuclear fragmentation. In conclusion, kenaf seed oils from the three extractions were cytotoxic towards $\mathrm{CaOV} 3$ cell line in a dose-dependent manner possibly via the induction of apoptosis. In considering the safety of the product, SFE technology is a better alternative extraction method that is suitable in kenaf seed oil extraction.
\end{abstract}

Keyword: Supercritical carbon dioxide fluid extraction; Kenaf (H. cannabinus) seed oil; Cytotoxicity; Apoptosis. 